\title{
Biosynthesis and Antimicrobial Activity Studies of Salicylalchitosan Functionalized Zinc Oxide Nanoparticles and Comparative Studies with its Non-Functionalized Form
}

\author{
M JAYANDRAN ${ }^{1}, M_{\text {MUHAMED HANEEFA }}^{2 *}$ and V BALASUBRAMANIAN ${ }^{2}$ \\ ${ }^{1} P G$ \& Research Department of Chemistry, Government Arts College, Trichy-22, India. \\ ${ }^{2}$ Department of Chemistry, AMET University, Chennai-112, India. \\ *Corresponding author: Email: honey79101@gmail.com
}

http://dx.doi.org/10.13005/ojc/320180

(Received: November 30, 2015; Accepted: January 30, 2016)

\begin{abstract}
Biofunctionalization of nanomaterial is one such topic which is an advancement of current nanotechnology and to produce the non-toxic and more efficient antimicrobial agents. It becomes incorporated for development of biosynthetic and eco-friendly technology for nanomaterials synthesis. Therefore, the present study describes a synthesis of bioactive salicylalchitosan functionalized zinc oxide nanoparticles through a biosynthesis method and comparative studies of those nanoparticles with zinc oxide nanoparticles were undertaken. The antimicrobial activities of non-functionalized and biofunctionalized zinc oxide nanoparticles were carried out against some well-known bacterial and fungal species. Synthesized materials were characterized by UV- Vis, FT-IR, SEM and TEM techniques and the results were reported.
\end{abstract}

Key words: Chitosan, Salicylalchitosan, Biosynthesis, Biofunctionalization, Antimicrobial activities.

\section{INTRODUCTION}

Biosynthesis of nanoparticles is a bottom up approach method where reduction/oxidation is the key reaction takes place. Plant extracts are rich sources of biomolecules, easily available, safe to handle and low cost which increases its part in the biosynthesis of nanoparticles. The microbial enzymes or the plant phytochemicals with antioxidant or reducing properties are usually the main reason for reduction of metal compounds into their respective nanoparticles ${ }^{1}$.

Biofunctionalization of nanoparticles can provide them with good biocompatibility for the immobilization of biomolecules and high specificity for biological recognition which led to produce a considerable effect in biological systems. Therefore, biofunctionalized nanomaterials are being given considerable attention in a multiple way of emerging 
fields of science and technology. However, pursuing appropriate biomaterials for the functionalization of nanomaterials is one of the crucial issues in this field. Various biomaterials were reported in the biosynthesis of nanoparticles ${ }^{2-3}$. Chitosan is a naturally occurring biopolymer derived from chitin. Chitosan is a cost-effective and renewable material with many applications in cosmetics, pharmaceuticals, food science and biotechnology ${ }^{4-5}$. It has been attracted more attention for its unique properties, biodegradable, flexibility, non-hazardous, easy availability and used as a carrier in drug delivery. It has important biological properties such as biocompatibility, antifungal and antibacterial activity, wound healing ability, anti-cancer property, anticholesteremic properties and immune enhancing effect $^{6-7}$.

Many investigations have been focused on metal nanoparticles particularly gold, silver and platinum concerning antimicrobial applications to develop new and effective antimicrobial reagents. Considering the higher cost associated with the above materials, researchers are trying to find an antimicrobial agents by using the low cost materials and methods. By considering this factor alternative metal and metal oxide nanoparticles such as copper, nickel, magnesium, iron etc. were focused and reported in literature ${ }^{8-9}$.

Zinc is an easily available and more reactive element and zinc nanoparticles are of great interest due to its essential constituent for cell growth and in inhibiting bacterial enzymes like dehydrogenase and certain protective enzymes such as thiolperoxidase and glutathione reductase. Various researches have shown that antimicrobial effect of $\mathrm{Zn}$ nanoparticles has been endorsed due to their good reactivity, which allows them to interact closely with microbial membranes. Zn nanoparticle acts efficiently to resist microorganisms and has a remarkable lengthy period of life than organic based disinfectants and antimicrobial agents ${ }^{10-12}$.

Based upon the above discussion, this investigation was carried out to synthesize cost effective and possess antimicrobial active zinc oxide nanoparticles which was biofunctionalized with salicylalchitosan to enhance its antimicrobial activity. Firstly, salicylalchitosan was synthesized from the chitosan then zinc oxide nanoparticles (ZONP) were prepared by reducing zinc acetate with the help of lemon extract. Finally, salicylalchitosan was functionalized with synthesized zinc oxide nanoparticles. The antimicrobial results of biofunctionalized zinc oxide nanoparticles (ZNSC) showed efficient inhibition activity against bacterial strains and funguses tested.

\section{MATERIALS AND METHODS}

The chemicals are of zinc acetate, aniline and ethanol were purchased from Merck (India) Ltd and all the chemicals and solvents used were of analytical reagent grade. Prawn shells of chitosan source was purchased from sea markets of Tuticorin, India. The biomaterial salicylalchitosan was prepared by using salicylaldehyde and chitosan in the laboratory ${ }^{13}$.

\section{Collection of extracts}

In this study fruits of citrus lemon were used as a bioreductant for the synthesis of zinc oxide nanoparticles. The main reason for selecting this, since lemons are a rich source of citric acid and ascorbic acid and it contains huge amounts of polyphenolic phytochemicals which is the excellent source for reducing agent ${ }^{14-15}$. Fresh lemon fruits were cut into pieces and squeezed well to get about $10 \mathrm{ml}$ pure extract. The extract was filtered by using whatman filter paper and the filtrate was collected and stored.

\section{Synthesis of salicylalchitosan (SC)}

According to Brine \& Austin, $1981^{16}$ Muzzarelli \& Jeuniaux, $1986^{17}$, the washed, dried and powdered prawn shells was de-mineralized with $1 \mathrm{M}$ $\mathrm{HCL}$ and deprotienized with $1 \mathrm{M}$ sodium hydroxide at $60^{\circ} \mathrm{C}$ with constant stirring for an hour. The pink coloured solution was washed several times and dried then deacetylated with $40 \%$ sodium hydroxide for 2 hours at $60^{\circ} \mathrm{C}$ and the obtained dirty white precipitate of chitosan was washed several times and dried in oven at $60^{\circ} \mathrm{C}$ for 3-4 hrs.

$100 \mathrm{mg}$ of dried chitosan powder was dissolved in $25 \mathrm{ml}$ of acetic acid solution (0.5 M) and kept on magnetic stirrer for $2 \mathrm{hrs}$. A mixture of 10 $\mathrm{ml}$ of salicylaldehyde $(0.1 \mathrm{M})$ added to the chitosan mixture then kept on hot magnetic stirrer at $50-60^{\circ} \mathrm{C}$ 
for 6 hours and refluxed well. The obtained yellowish green precipitate of salicylalchitosan was washed well and dried under vacuum at $60^{\circ} \mathrm{C}$ for $12 \mathrm{hrs}$.

\section{Synthesis of zinc oxide nanoparticles (ZONP)}

The lemon extract $(10 \mathrm{ml})$ was added drop wise to the zinc salt $(1 \mathrm{mmol})$ solution and kept in magnetic hot stirrer at $50-60^{\circ} \mathrm{C}$ for an hour and the colour gets changed from pale green to colourless which indicated the metal ion reduction. Upon the addition of curcumin extract $(1 \mathrm{mmol})$, the colourless solution instantly turned yellow and the hot stirring was continued for about 2 hours. The reaction mixture colour was changed to yellowish brown and finally a permanent brown colour which implied that complete stabilized ZONP. The reaction $\mathrm{pH}$ and temperature were maintained in between 3 to 4 and $50-60^{\circ} \mathrm{C}$ respectively throughout the experiment. The mixture was centrifuged and washed well to obtain the pure ZONP. The supernatant was decanted and dried in oven to use further analyses.

\section{Synthesis of biofunctionalized zinc oxide nanoparticles (ZNSC)}

In a typical synthesis of biofunctionalized zinc oxide nanoparticles, functionalization of salicyalalchitosan with zinc oxide nanoparticles was carried out under constant stirring and heating at $60^{\circ} \mathrm{C}$. The above synthesized $1 \mathrm{mmol}$ of ZONP was mixed with $1 \mathrm{mmol}$ of salicylalchitosan and kept on hot magnetic stirrer for an hour. The mixture color was turned to change from brown colour to dark brown which denoted the salicylalchitosan functionalized zinc oxide nanoparticles. The solution was centrifuged and the upper layer was removed and dried for further analyses.

\section{Biological Assay}

The antibacterial activity of the samples were tested against two gram positive bacteria (Staphylococcus aureus and Bacillus subtilis), two gram negative bacteria (Escherichia coli and Staphylococcus bacillus) and antifungal activity was carried out against four funguses (Candida albicans, Curvularia lunata, Aspergillus nigerand Trichophyton simii).

The antibacterial activity of samples were measured by disc diffusion method ${ }^{18}$. In this method, the stock cultures were incubated in nutrient agar and transferred to Muller-Hinton broth (MHB) contained test tube for bacteria that were incubated for 24 hours at $37^{\circ} \mathrm{C}$. The cultures were diluted with fresh Muller-Hinton broth to obtain $2.0 \times 10^{6} \mathrm{CFU} / \mathrm{ml}$ for bacteria. The Muller Hinton Agar (MHA) plates were prepared by pouring $15 \mathrm{ml}$ of molten media into sterile petri plates. The sample was loaded on the surface of the cultured agar plates and incubated at $37^{\circ} \mathrm{C}$ for 24 hours. The inhibition zones observed around the disc were measured.

The antifungal activity of samples were measured by agar well diffusion method ${ }^{19}$, the fungal strains were suspended in sabouraud's dextrose broth for 6 hours to give concentration $10^{5} \mathrm{CFU} /$ $\mathrm{ml}$ and then inoculated with the culture medium. A total of $8 \mathrm{~mm}$ diameter wells were punched into the agar and filled with the sample and solvent blanks (hydro alcohol and hexane). Standard antibiotic, Fluconazole (concentration $1 \mathrm{mg} / \mathrm{ml}$ ) was used as positive control and fungal plates were incubated at $37^{\circ} \mathrm{C}$ for 72 hours. The diameters of zone of inhibition observed were measured.

\section{RESULTS AND DISCUSSION}

The UV-Visible absorption spectra of the samples were measured on a Shimadzu UV-Vis $\mathrm{V}-530 \mathrm{~A}$ spectrophotometer in the range of 300 to $900 \mathrm{~nm}$. Figure 1 and 2 shows a UV spectrum of non-functionalized and biofunctionalized zinc oxide nanoparticles respectively. The UV spectra of ZONP exhibited a peak at $300 \mathrm{~nm}$ which belongs to the absorption of zinc oxide nanoparticles (Figure 1). In the case of ZNSC, the same zinc oxide nanoparticles absorption peak was observed at $301 \mathrm{~nm}$ and next to this, there was a broaden peak has been observed at $392 \mathrm{~nm}$ which could be due to the functionalization of salicylalchitosan with zinc oxide nanoparticles (Figure 2). The other peak observed in lower wavelength in both formulations at around $225 \mathrm{~nm}$ could be due to the presence of aggregation of ZONP during the nanoparticle synthesis.

FT-IR spectra analysis was recorded on a Jasso FT-IR/4100 spectrophotometer with $4 \mathrm{~cm}^{-1}$ resolution in the range of 4000 to 400 $\mathrm{cm}^{-1}$. Figure 3 shows the FT-IR spectrum of zinc oxide nanoparticles (Bottom) and functionalized nanoparticles (Top). ZONP showed the following 
important peaks at different regions, $3389 \mathrm{~cm}^{-1}$ (ph$\mathrm{OH}$ of curcumin), $2926 \mathrm{~cm}^{-1}$ (C-H stretching of water or ethanol), $1704 \mathrm{~cm}^{-1}$ ( $\mathrm{C}=\mathrm{O}$ stretching of curcumin), $1393 \mathrm{~cm}^{-1}$ (C=C stretching of curcumin), 1000-1250 $\mathrm{cm}^{-1}$ (C-O stretching-3 bands). In the case of ZNSC, the observed important peaks are at $3390 \mathrm{~cm}^{-1}(\mathrm{O}-\mathrm{H}$ polymeric stretching of chitosan), $1703 \mathrm{~cm}^{-1}(\mathrm{C}=\mathrm{O}$ stretching of SC), $1574 \mathrm{~cm}^{-1}$ and $1557 \mathrm{~cm}^{-1}$ (C=C aromatic ring stretching), $1385 \mathrm{~cm}^{-1}(\mathrm{O}-\mathrm{H}$ stretching of ph-OHCHO coordinated with chitosan), $1179 \mathrm{~cm}^{-1}$ (C-N stretching of tertiary amine of chitosan), 1032 $\mathrm{cm}^{-1}(\mathrm{C}-\mathrm{N}$ stretching of $\mathrm{CH}=\mathrm{N}$ present in $\mathrm{SC}$ ), 800$900 \mathrm{~cm}^{-1}$ (C-O band).

Scanning electron microscopy (SEM) images were recorded by using JEOL Model JSM - 6390LV scanning electron microscope. The SEM images of ZONP and ZNSC were shown in Figure $4 a$ and $4 b$ respectively. It can be view that ZONP shown rod and spherical shaped crystals morphology of material (Figure 4a). In the case of ZNSC, the image exhibited slightly agglomerated and dispersed

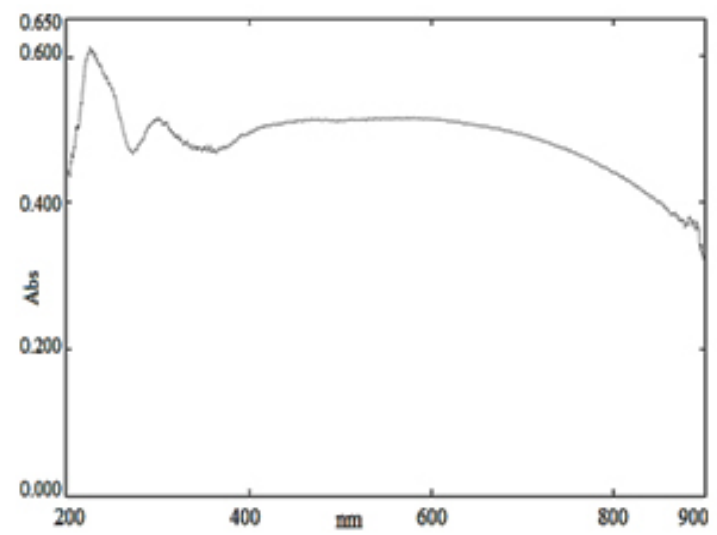

Fig. 1: UV spectra of ZONP spherical shaped crystals morphology due to the nanoparticles oxidation (Figure 4b).

High resolution transmission electron microscopy (HRTEM) was carried out using a 300 KV JEOL-3011 instrument to determine the size and morphological changes. Figure $5 \mathrm{a}$ and $5 \mathrm{~b}$ shows the TEM images of the non-functionalized and salicylalchitosan functionalized zinc oxide nanoparticles respectively. ZONP are slightly agglomerated and average crystallite size of particles is in the range of 23-27 nm with different shaped morphology (Figure 5a). ZNSC exhibits moderately dispersed spherical morphology and the average crystallite size of particles are in the range of $46-50 \mathrm{~nm}$. The size increment may due to the functionalization of salicylalchitosan with ZONP (Figure 5b).

\section{Antibacterial Activity}

The antibacterial activity of biologically produced nanoparticles were tested on different

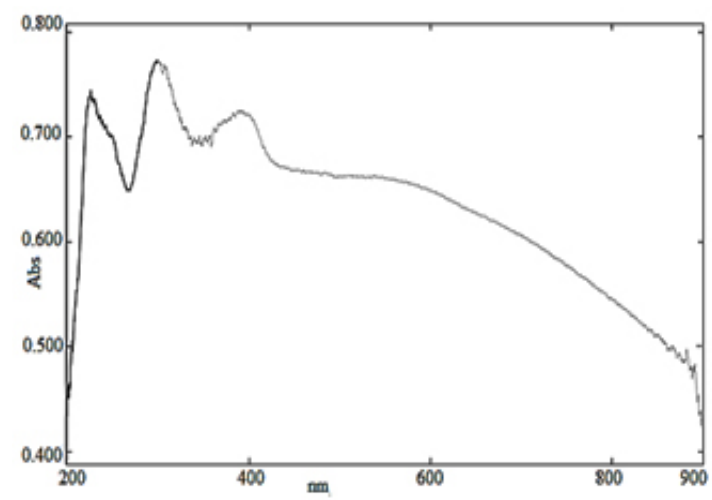

Fig. 2: UV-Vis spectrum of ZNSC

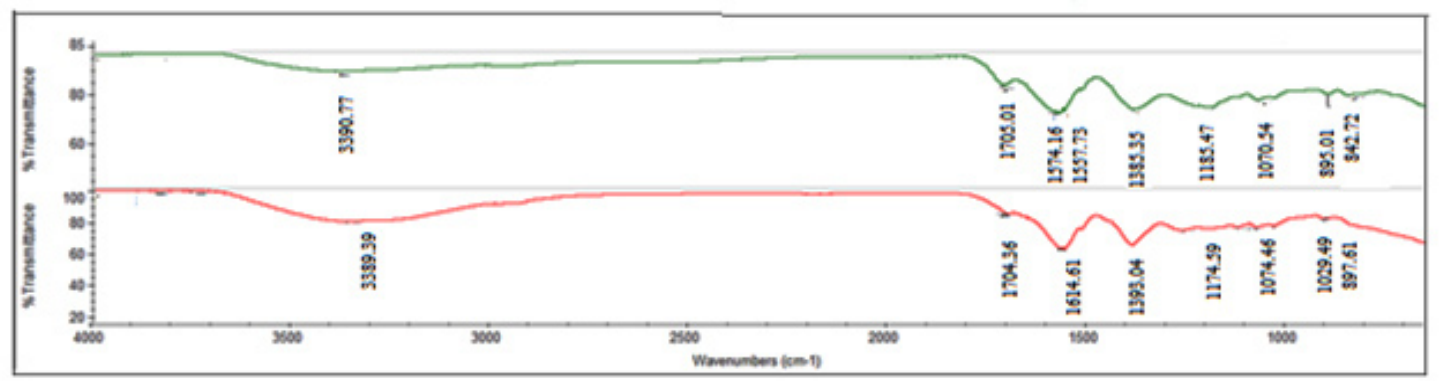

Fig. 3: IR spectra of ZONP (Bottom); ZNSC (Top) 
bacteria Staphylococcus aureus, Bacillus subtilis, Escherichia coli and Staphylococcus bacillus. The inhibition tests were compared to standard drug, Chloramphenicol. The results are reported in Table 1.

From Table 1, the results of biofunctionalized zinc oxide nanoparticles shown better antibacterial activity than zinc oxide nanoparticles. Nonfunctionalized ZONP shown reasonable inhibition activity against S.aureus, B.subtilis and S.bacillus species. But ZNSC displayed an appreciable inhibition activity than ZONP against the above

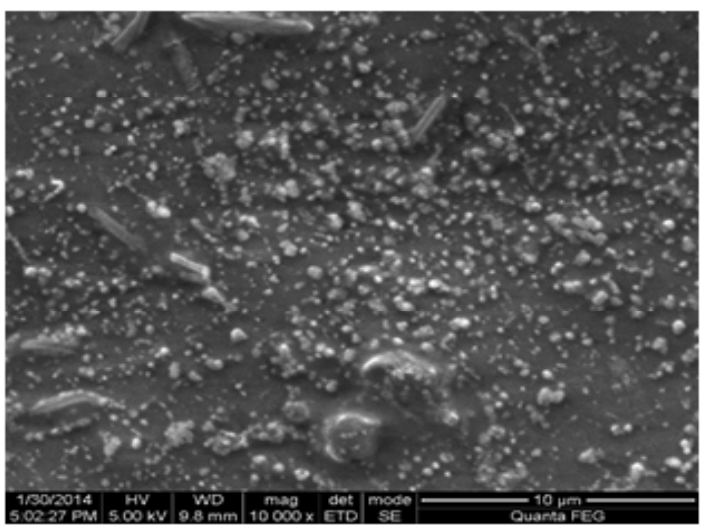

species. It showed similar activity to ZONP against S.bacillus and lower activity against E.coli only. Especially, it showed higher inhibition zone results than standard drug, Chloramphenicol against S.aureus and B.subtilis strains. The difference observed in the activity enhancement is due to the biomolecule functionalization which increased the antimicrobial efficiency to the nanoparticles.

\section{Antifungal Activity}

ZONP and ZNSC were determined for their antifungal activity against four fungal strains Candida albicans, Curvularia lunata, Aspergillus

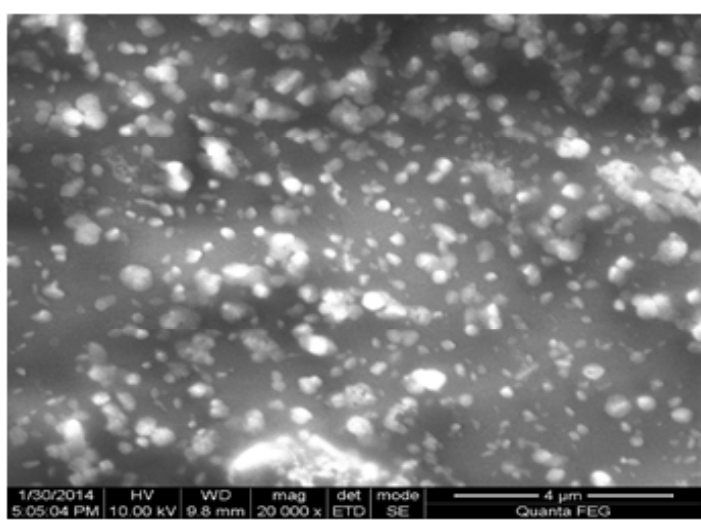

Fig. 4: SEM images of ZONP (a); ZNSC (b)

Table 1: Antibacterial activity evaluation

\begin{tabular}{|c|c|c|c|}
\hline \multirow{2}{*}{$\begin{array}{l}\text { Bacterial } \\
\text { Species }\end{array}$} & \multicolumn{3}{|c|}{ Zone of inhibition diameter (mm sample ${ }^{-1}$ ) } \\
\hline & $\begin{array}{l}\text { Standard } \\
\text { drug }(C)\end{array}$ & $\begin{array}{c}\text { Zinc oxide } \\
\text { nanoparticles (ZONP) }\end{array}$ & $\begin{array}{l}\text { Biofunctionalized zinc oxide } \\
\text { nanoparticles (ZNSC) }\end{array}$ \\
\hline S. aureus & 13 & 14 & 20 \\
\hline B.subtilis & 15 & 15 & 16 \\
\hline E. coli & 11 & 08 & 06 \\
\hline S.bacillus & 14 & 13 & 13 \\
\hline
\end{tabular}

Table 2: Antifungal activity evaluation

\begin{tabular}{lccc}
\hline $\begin{array}{l}\text { Fungal } \\
\text { Species }\end{array}$ & \multicolumn{2}{c}{$\begin{array}{c}\text { Zone of inhibition diameter (mm sample }{ }^{-1} \text { ) } \\
\text { Standard } \\
\text { drug (C) }\end{array}$} & $\begin{array}{c}\text { Zinc oxide } \\
\text { nanoparticles (ZONP) } \\
\text { nanctionalized zinc oxide } \\
\text { nanoparticles (ZNSC) }\end{array}$ \\
\hline C.albicans & 16 & 12 & 19 \\
C.lunata & 15 & 13 & 13 \\
A.niger & 15 & 17 & 17 \\
T.simii & 13 & 15 & 20 \\
\hline
\end{tabular}



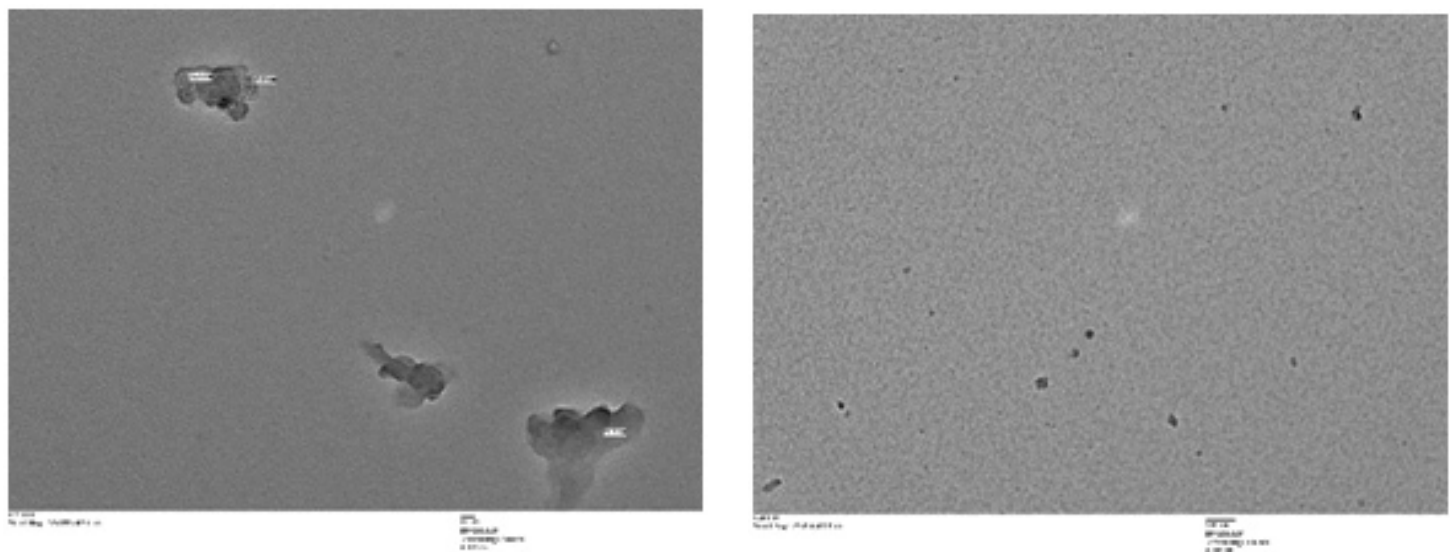

Fig. 5: TEM images of ZONP (a); ZNSC (b)

niger and Trichophyton simii and their activity was compared with standard antifungal drug Fluconazole. The results were shown in Table 2 .

From Table 2, the results revealed that biofunctionalized zinc oxide nanoparticles observed higher antifungal activity than non-functionalized zinc oxide nanoparticles. ZONP showed similar activity to ZNSC against A.niger and C.lunata fungal strains, however, the inhibition zone results observed by ZNSC were higher than ZONP as well as standard drug, Fluconazole against C.albicans and T.simii fungal species and the overall antifungal activity of ZNSC were more appreciable. Therefore, functionalization of salicylalchitosan is the important factor in the result of increment.

In summary, this investigation has been carried out to synthesize salicylalchitosan functionalized zinc oxide nanoparticles by interacting zinc oxide nanoparticles with salicylalchitosan. The antimicrobial activity studies expressed that biofunctionalized nanoparticles were showed better activity than the non-functionalized nanoparticles against S.aureus, B.subtilis, C.albicans and T.simii. The morphology studies were shown that ZONP size was about 23-27 nm and ZNSC was in the range of 46-50 $\mathrm{nm}$. This may due to the biofunctionalization, however, there was an increment of antimicrobial activity only observed in the antimicrobial tests. Particularly, ZNSC shown higher activity than the standard drug against S.aureus, B.subtilis, C.albicans and T.simii. Therefore, our report reveals that biofunctionalization of salicylalchitosan increases the antimicrobial activity of zinc oxide nanoparticles which gives the new idea in the biomaterial utilized nanoparticle preparation.

\section{ACKNOWLEDGEMENTS}

We thank AMET University, Chennai, India for their support to do this work. We gratefully acknowledge Advanced Instrumentation Research Facility, Jawaharlal Nehru University, New Delhi for TEM analysis, Nanotechnology Research Centre, SRM University, Chennai for SEM analysis.

\section{REFERENCES}

1. Kavitha, K.S.; Syed Baker.; Rakshith, D.; Kavitha, H.U.; Yashwantha Rao, H.C.; Harini, B.P.; Satish, S. International Research Journal of Biological Sciences. 2013, 2, 66-76.

2. Challa Kumar, S.S.R. Biological and pharmaceutical nanomaterials, Vol. II.; WileyVCH Verlag GmbH Co. KGaA. Weinheim, (2005).
3. Goesmann, H.; Feldmann, C. Angew. Chem. Int. Ed. 2010, 49, 1362-1395.

4. Katz, E.;Willner, I. Angew. Chem. Int. Ed. 2004, 43, 6042-6108.

5. Kim, S. J.; Shin, S. R.; Lee, Y. M.; Kim, S. I. J. Appl. Polym. Sci. 2003, 87, 2011-2015.

6. Lei, C. X.; Hu, S. Q.; Shen, G. L.; Yu, R.Q. Talanta, 2003, 59, 981-993. 
7. Reem K. Farag.; and Riham R. Mohamed. Molecules, 2013, 18, 190-203.

8. Jeong, S.; Woo, K.; Kim, D.; Lim, S.; Kim, J. S.; Adv Funct Mater. 2008, 18, 679-686.

9. Stoimenov, P. K.; Klinger, R. L.; Marchin, G.L.; Glebunde, K. Lang. 2002, 18, 6679-6686.

10. Narayanan, R.; and El-Sayed, M. A. J. Am. Chem. Soc. 2003, 125, 8340-8347.

11. Brayner, R.; Ferrari-lliou, R.; Brivois, N.; Djediat, S. Nano Lett. 2006, 6, 866-870.

12. Gajjar, P.; Pettee, B.; Britt, D. W.; Huang, W.; Johnson, W. P.; Anderson, A. J. Journal of Biological Engineering. 2009, 3, 1-13.
13. Jayandran, M.; Muhamed haneefa, M. Chem. Sci. Rev. Lett. 2014, 3, 1050-1059.

14. Limer, J. L.; and Speirs, V. Breast Cancer Res. 2004, 6, 119-127.

15. Bandele, O. J.; and Osheroff, N. Chem Res Toxicol. 2008, 21, 936-43.

16. Charles J. Brine.; Paul R. Austin. Comparative Biochemistry and Physiology Part B: Comparative Biochemistry, 1981, 70, 173178.

17. Muzzarelli, R. A. A.; Jeuniaux, C.; Gooday, G. W. (Eds). Chitin in Nature and Technology, Plenum. New York, USA, (1986). 